\title{
Bartonella Infection in Immunocompromised Hosts: Immunology of Vascular Infection and Vasoproliferation
}

\author{
Mosepele Mosepele, Dana Mazo, and Jennifer Cohn \\ Department of Medicine, Hospital of the University of Pennsylvania, 100 Centrex, Philadelphia, PA 19104, USA \\ Correspondence should be addressed to Mosepele Mosepele, mosepele.mosepele@uphs.upenn.edu
}

Received 15 July 2011; Revised 27 September 2011; Accepted 29 September 2011

Academic Editor: Georgios Pappas

Copyright ( $) 2012$ Mosepele Mosepele et al. This is an open access article distributed under the Creative Commons Attribution License, which permits unrestricted use, distribution, and reproduction in any medium, provided the original work is properly cited.

\begin{abstract}
Most infections by genus Bartonella in immunocompromised patients are caused by $B$. henselae and B. quintana. Unlike immunocompetent hosts who usually develop milder diseases such as cat scratch disease and trench fever, immunocompromised patients, including those living with HIV/AIDS and posttransplant patients, are more likely to develop different and severe lifethreatening disease. This paper will discuss Bartonella's manifestations in immunosuppressed patients and will examine Bartonella's interaction with the immune system including its mechanisms of establishing infection and immune escape. Gaps in current understanding of the immunology of Bartonella infection in immunocompromised hosts will be highlighted.
\end{abstract}

\section{Introduction}

In this paper, we present a summary of the basic characteristics of Bartonella species which are important etiologic agents in human disease. The discussion focuses on a review of what is known of the immunology of Bartonella, particularly in immunocompromised hosts.

\section{Classification}

Bartonella are fastidious hemotropic facultative intracellular Gram-negative bacteria of the alpha-2 subgroup of Proteobacteria $[1,2]$. Of the 24 species that comprise the genus Bartonella, B. bacilliformis, B. henselae, and B. quintana infections have been most commonly implicated in human disease and $B$. henselae and $B$. quintana account for the majority of morbidity and mortality among immunocompromised individuals [3-5].

\section{Bartonella Epidemiology}

Each of the major Bartonella species implicated in human disease has unique epidemiology. While the vast majority of Bartonella species have an identified zoonotic reservoir such as domesticated animals and farm animals, the animal reservoir for both $B$. quintana and $B$. bacilliformis have not yet been identified. While $B$. bacilliformis is transmitted by the sand fly and the major vector for $B$ quintana is the human body louse, $B$ quintana has also been found in rodent fleas, including rat fleas, and thus a potential for a rodent vector has been hypothesized $[6,7]$.

$B$. henselae occurs throughout the world. It is a zoonosis transmitted from the natural reservoir, cats (Felis catus), usually via cat scratch or bite and less commonly by a vector such as cat fleas (Ctenocephalides felis) or ticks [8]. Crosssectional studies have reported as much as $81 \%$ seropositivity point prevalence among cats in different regions of the world. Bacteremia is more prevalent in cats that are less than 1 year old [2]. Humans and dogs are accidental hosts.

Although B. quintana occurs worldwide, with improved sanitation and living conditions, disease caused by B. quintana has dramatically decreased and now occurs primarily in small epidemics in conditions characterized by crowding and poor sanitation.

Humans are likely the natural reservoir for B. quintana which is spread by human body lice (Pediculus humanis corporis) [8]. Infected body lice excreta on human skin induce pruritis, and skin breakdown that follows scratching allows for B. quintana's pathway of invasion [9]. Wild rodents have also been identified as potential reservoirs $[6,7]$. 
B. bacilliformis is endemic in mountainous areas of Peru, Columbia, and Ecuador [10]. It is transmitted by the Lutzomyia sandfly, but the animal reservoir has yet to be identified.

\section{Bartonella and the Human Immune System}

4.1. Establishing Infection: Evasion of the Immune System. Despite extensive knowledge about the disease syndromes caused by Bartonella species in humans, little is known about the humoral and cellular immune mechanisms involved in establishment and persistence of Bartonella infection in humans. Experimental studies using B. tribocorum inoculation onto an immune-competent rat model have shown that the brief bacteremia after inoculation is followed by a 3-5day bacteremia-free window before resurgence of persistent intraerythrocytic bacteremia that lasts for 8-11 weeks [11]. This model highlights possible existence of a sanctuary site where the initial infection gets established before persistent intraerythrocytic infection manifests. So far, three such potential sanctuary sites include (a) extracellular matrix where high local bacterial replication may be achieved following initial infection [11], (b) bone-marrow stem cells such as erythroid progenitor cells where CD34+ cells in the bone marrow may be infected followed by release of infected erythrocytes into the blood stream [12] or endothelial progenitor cells which may also get infected in the bone marrow prior to their mobilization into circulation for endothelial repair [13], and, finally, (c) mature endothelial cells [14].

Bartonella Has Several Characteristics That Lead to Immune Evasion. Bartonella lipopolysaccaride (LPS) is composed of a unique combination of Lipid $\mathrm{A}$ and long-chain fatty acids and likely contributes to the bacteria's ability to evade the immune system [15]. Empiric data have shown that Bartonella initially evades the innate immune system because its surface molecules are not recognized by TLR-4 on dendritic cells or macrophages, [16], thereby allowing for establishment of what might lead to persistent infection. The LPS does not induce tumor necrosis factor alpha and has reduced stimulation of Toll-like receptor 2 (TLR2) and thus reduced endotoxicity $[17,18]$. The LPS of $B$. quintana may even antagonize the Toll-like receptor 4 (TLR4) [19], which is a key component of innate immunity. Popa et al. and Matera et al. demonstrated that LPS from B. quintana resulted in the downregulation in human monocytes of nearly all cytokines normally produced by TLR4 in response to LPS $[19,20]$ and B. quintana's antagonism of TLR4 may be responsible for the absence of symptoms normally associated with bacteremia with Gram-negative organisms, such as septic shock.

Furthermore, $B$. henselae can avoid lysosomal fusion and acidification after the bacteria invades phagocytes such as endothelial cells and macrophages. For instance, $B$. henselae containing vacuoles (BCVs) had delayed development or complete lack of typical endocytic markers [21]. When the BCV contained inert or killed B. henselae, the endocytic markers were in normal ranges. Such Dendritic cells within cat scratch disease (CSD) granuloma in immune-competent humans that are chronically infected with Bartonella are also able to limit the infection to the same lymph node region via a localized B-cell-mediated humoral immune activation that is regulated by dendritic cells [22]. On the contrary, immunocompromised persons develop bacteremia, and distant seeding may occur, hence recovery of Bartonella in cardiac valves, other vasoproliferative lesions (bacillary angiomatosis or peliosis). Exactly how local bacterial control is lost in immunocompromised subjects is unclear.

4.2. Bacterial Persistence: Pro- and Anti-Inflammatory Response. Historically, clinical and in vitro studies implicated a Th1 immune response to Bartonella infection. The old Bartonella skin test involved an intradermal injection of suppurative material derived from lymph nodes from cat scratch disease patients and was interpreted as positive if their skin developed a large erythematous area within $48 \mathrm{hrs}$. This test is a classic delayed type hypersensitivity reaction, mediated through the Th1 immune response. In vitro studies have also supported the use of a Th1 response with mice splenocytes showing increased production of the Th1 cytokines IFN $\gamma$ and IL-12 in response to B. henselea when compared with controls [23]. The role of innate immunity is also demonstrated through the repeated evidence of elevated IL-8 in response to $B$. henselae [24].

More recent studies have focused on the role of cytokines in acute and chronic Bartonella infection. Acute infection in immune-competent subjects with CSD have revealed upregulation of proinflammatory (IL-2, IL-6) and antiinflammatory (IL-10) cytokines [25]. On the contrary, low CD4 counts (a marker of immune-compromise) have been associated with elevated IL-10 levels during acute infection with Bartonella, a cytokine milieu that may allow an acute infection to persist especially given the anti-inflammatory nature of IL-10 $[16,25,26]$. Chronic infection is associated with elevated levels of Interferon-alfa and IL- 4 in both animal $[16,27]$ and human studies of the bacillary angiomatosis [16], but not in immune-competent subjects with CSD [25]. Thus, far, empiric data regarding Bartonella infection persistence and role in vasoproliferation is better understood from cytokine profiles, and less so from the traditional roles of humoral or cellular immunity.

Most of the research in infection involving immunocompromised hosts has focused on the establishment of bacterial stimulated angiogenesis. Understanding of the immune defense of Bartonella species in immunocompromised patients has been hampered by the lack of good animal model [28]. Since the response in the competent immune system involves Th1 and innate immunity through macrophages, a logical inference can be made that HIVpositive and other patients deficient in these immunological areas would have difficulty limiting the infection and thus would develop systemic manifestations. This deduction, however, has not yet been demonstrated as the mechanism by which HIV facilitates the spread of Bartonella. Chiaraviglio et al. described a murine model for chronic Bartonella infection using B. taylorii in SCID/Beige mice (B-, T-, NKcell deficient) [29]. The group described that this model 
approximated human bacillary peliosis and splenitis through Bartonella growing in extracellular aggregates in the spleen and liver. As the model is still relatively new, they did not present any further characterization of the immune response. However, it is clear that intraerythrocytic persistence is characteristic of infections in immune-competent hosts while endothelial/periendothelial persistence leading to vasoproliferation is characteristic of an infection in immunecompromised hosts [29].

\section{Pathophysiology of Bartonella Disease/Lesions in Immunocompromised Hosts}

5.1. Angiogenesis. Bacillary angiomatosis is the most common sequelae of $B$. quintana and $B$. henselae infections in patients with cell-mediated immunodeficiency such as HIV and posttransplant patients on immunosuppressive therapy $[9,23,30] . B$. henselae and $B$. quintana induce their characteristic vasoproliferative lesion, bacillary angiomatosis, or peliosis, in immunosuppressed patients, by direct and indirect effects on endothelial cells.

Whereas most pathogenic bacteria achieve tissue destruction and wider dissemination by inducing apoptosis on host cells, $B$. henselae and $B$. quintana infections persist within periendothelial extracellular matrix resulting in sustained, localized bacterial replication within collagen tissue [29]. This localized bacterial replication facilitates an antiapoptotic state in endothelial cells by secreting effector proteins (BepA and BepA2) which bind to the endothelial membrane receptor. The ensuing transmembrane signal transduction results in high cytoplasmic cAMP levels. The high cAMP levels upregulate cAMP responsive genes and induce an antiapoptotic state in the endothelial cells, resulting in their proliferation [31]. In addition, Schmid et al. in the same experiment showed that BepA can inhibit cytotoxic T-cellmediated apoptosis of endothelial cells infected by $B$. henselae and $B$. quintana. A further analysis of the antiapoptotic mechanisms of some $B$. henselae strains isolated from HIVinfected patients revealed that an anti-apoptotic state may be effected through $B$. henselae strain-specific upregulation of antiapoptotic or downregulation of proapoptotic factors that work through the mitochondrial intrinsic apoptotic pathway [32]. As noted earlier, Interferon-alfa and IL-4 in chronic Bartonella infections may play a role, albeit unclear, in the development of bacillary angiomatosis given that elevated levels have been reported both animal [27] and human studies of the bacillary angiomatsois [25], but not in immune-competent subjects with CSD [25].

$\mathrm{NF}-\kappa \mathrm{B}$ is also upregulated by Bartonella, resulting in increased leukocyte rolling and adhesion and causes a proinflammatory environment [12]. Additional research is needed to explore this link between some $B$. henselae strains that infect HIV patients and vasoproliferation and inflammation. Such work may shed more light into pathogenicity of $B$. henselae and host susceptibility factors in HIV-positive patients.

\section{Immunocompromise Induced by Bartonella Infections}

B. bacilliformis itself induced an immunocompromised state. Although B. bacilliformis is not widespread in distribution and has not been described as a cause of bacillary angiomatosis or hepatic peliosis in immunocompromised patients, it is notable that the acute bacteremic phase of B. bacilliformis causes significant immunosuppression by overloading the reticuloendothelial system. These patients are predisposed to infections such as salmonella and tuberculosis [28].

\section{Clinical Features of Bartonella Infections in Immunocompromised Hosts}

Bartonella infections caused by $B$. henselae or B. quintana in the immunocompromised host may be characterized by fever of unknown origin, culture-negative endocarditis, osteomyelitis, or angioproliferative lesions that may affect virtually any organ system, but have a predilection for the skin, liver, and spleen.

7.1. Clinical Features of Bartonella Infections in HIV-Infected Patients. Bacillary angiomatosis lesions and fever are the most common manifestation of $B$. henselae or B. quintana infection in HIV/AIDS patients [3, 9]. The lesions appear as cutaneous nodular vascular lesions but may also be found in a variety of organs including the GI-tract where they may cause hematemesis [33-35], genitourinary system [36], and other organs including heart, spleen, bones, and central nervous system. The differential diagnosis for bacillary angiomatosis includes Kaposi's sarcoma, angiosarcoma, and pyogenic granuloma.

Similar vascular lesions are seen in both bacillary peliosis and splenitis. Patients present with hepato- and/or splenomegaly as blood filled cysts proliferate in these organs.

Confirming the diagnosis can be challenging as serology has a $50-95 \%$ sensitivity and demonstrates cross-reaction between Bartonella, Coxiella, and Chlamydia species. Results from tissue culture are not always positive and take a long time given the fastidious nature of the bacterium. PCR is favored as a faster, sensitive, and specific diagnostic test $[37,38]$.

7.2. Clinical Features of Bartonella Infections in Transplant Patients. Persistent fever following close contact with cats is the most common presenting symptom among posttransplant patients who are infected with $B$. henselae [39]. $B$. henselae infection was complicated by acute graft rejection in 2 renal transplant patients [40]. It is unclear if this rejection was a random occurrence or if it is associated with some unknown effect of $B$. henselae on decreasing the donor tolerance of the graft or enhancing graft antigenicity. In liver transplant patients, $B$. henselae can present with hepatic masses [41], hepatic granulomas [42, 43], or disseminated disease [44].

7.3. Clinical Features of Bartonella Infection in Homeless Patients with Chronic Alcoholism. Subacute, culture-negative 
endocarditis is the most common clinical presentation of $B$. quintana (less so $B$. henselae) infection among homeless patients with chronic alcoholism $[4,45,46]$. Risk factors for Bartonella endocarditis include preexisting valvular lesions and exposure to human body lice.

B. quintana may be cultured from the blood, but given the prolonged time required for culture, other diagnostic tests are preferred. An indirect immunofluorescent IgG antibody test directed against Bartonella species with titer of $1: 800$ or higher has a $95 \%$ positive predictive value for diagnosing Bartonella endocarditis [47]. Diagnosis is usually confirmed by performing PCR testing on plasma or tissue samples following DNA extraction [6].

\section{Conclusion}

Bartonella species are important zoonotic agents of human disease. Immunocompromised hosts are more susceptible to infection from $B$. henselae or B. quintana which results in a more severe clinical picture as compared with the normal host. More recent work explores role of cytokines and chemokines in subverting the immune system, and subsequently a persistent infection characterized by vasoproliferation in immunocompromised humans. The mechanisms of establishing infection and immune evasion in immunocompromised hosts are still incompletely understood and are areas where further studies are needed.

\section{References}

[1] M. Maurin and D. Raoult, "Bartonella infections: diagnostic and management issues," Current Opinion in Infectious Diseases, vol. 11, no. 2, pp. 189-193, 1998.

[2] B. B. Chomel, R. C. Abbott, R. W. Kasten et al., "Bartonella henselae prevalence in domestic cats in California: risk factors and association between bacteremia and antibody titers," Journal of Clinical Microbiology, vol. 33, no. 9, pp. 2445-2450, 1995.

[3] J. E. Koehler, M. A. Sanchez, S. Tye et al., "Prevalence of Bartonella infection among human immunodeficiency virusinfected patients with fever," Clinical Infectious Diseases, vol. 37, no. 4, pp. 559-566, 2003.

[4] D. Raoult, P. E. Fournier, M. Drancourt et al., "Diagnosis of 22 new cases of Bartonella endocarditis," Annals of Internal Medicine, vol. 125, no. 8, pp. 646-652, 1996.

[5] P. E. Fournier, H. Lelievre, S. J. Eykyn et al., "Epidemiologic and clinical characteristics of Bartonella quintana and Bartonella henselae endocarditis: a study of 48 patients," Medicine, vol. 80 , no. 4 , pp. 245-251, 2001.

[6] K. J. Bown, M. Bennett, and M. Begon, "Flea-borne Bartonella grahamii and Bartonella taylorii in bank Voles," Emerging Infectious Diseases, vol. 10, no. 4, pp. 684-687, 2004.

[7] J. L. Marié, P. É. Fournier, J. M. Rolain, S. Briolant, B. Davoust, and D. Raoult, "Molecular detection of Bartonella quintana, B. elizabethae, B. koehlerae, B. doshiae, B. taylorii, and Rickettsia felis in rodent fleas collected in Kabul, Afghanistan," American Journal of Tropical Medicine and Hygiene, vol. 74, no. 3, pp. 436-439, 2006.

[8] B. B. Chomel, H. J. Boulouis, and E. B. Breitschwerdt, "Cat scratch disease and other zoonotic Bartonella infections," Journal of the American Veterinary Medical Association, vol. 224, no. 8, pp. 1270-1279, 2004.
[9] D. Raoult and V. Roux, "The body louse as a vector of reemerging human diseases," Clinical Infectious Diseases, vol. 29, no. 4, pp. 888-911, 1999.

[10] C. Maguiña and E. Gotuzzo, "Bartonellosis: new and old," Infectious Disease Clinics of North America, vol. 14, no. 1, pp. 1-22, 2000.

[11] J. Koesling, T. Aebischer, C. Falch, R. Schülein, and C. Dehio, "Cutting edge: antibody-mediated cessation of hemotropic infection by the intraerythrocytic mouse pathogen Bartonella grahamii," Journal of Immunology, vol. 167, no. 1, pp. 11-14, 2001.

[12] O. Fuhrmann, M. Arvand, A. Göhler et al., "Bartonella henselae induces NF- $\kappa \mathrm{B}$-dependent upregulation of adhesion molecules in cultured human endothelial cells: possible role of outer membrane proteins as pathogenic factors," Infection and Immunity, vol. 69, no. 8, pp. 5088-5097, 2001.

[13] P. Salvatore, A. Casamassimi, L. Sommese et al., "Detrimental effects of Bartonella henselae are counteracted by L-arginine and nitric oxide in human endothelial progenitor cells," Proceedings of the National Academy of Sciences of the United States of America, vol. 105, no. 27, pp. 9427-9432, 2008.

[14] C. Dehio, "Bartonella interactions with endothelial cells and erythrocytes," Trends in Microbiology, vol. 9, no. 6, pp. 279$285,2001$.

[15] M. W. Hornef, M. J. Wick, M. Rhen, and S. Normark, "Bacterial strategies for overcoming host innate and adaptive immune responses," Nature Immunology, vol. 3, no. 11, pp. 1033-1040, 2002.

[16] E. Huarcaya, I. Best, J. Rodriguez-Tafur et al., "Cytokines and T-cell lymphocytes count in patients in the acute and chronic phases of Bartonella bacilliformis infection in an endemic area in Peru: a pilot study," Revista do Instituto de Medicina Tropical de Sao Paulo, vol. 53, no. 3, pp. 149-154, 2011.

[17] G. Matera, M. C. Liberto, A. Quirino et al., "Bartonella quintana lipopolysaccharide effects on leukocytes, CXC chemokines and apoptosis: a study on the human whole blood and a rat model," International Immunopharmacology, vol. 3, no. 6, pp. 853-864, 2003.

[18] U. Zähringer, B. Lindner, Y. A. Knirel et al., "Structure and biological activity of the short-chain lipopolysaccharide from Bartonella henselae ATCC 49882T," Journal of Biological Chemistry, vol. 279, no. 20, pp. 21046-21054, 2004.

[19] C. Popa, S. Abdollahi-Roodsaz, L. A. B. Joosten et al., "Bartonella quintana lipopolysaccharide is a natural antagonist of toll-like receptor 4," Infection and Immunity, vol. 75, no. 10, pp. 4831-4837, 2007.

[20] G. Matera, M. C. Liberto, L. A. B. Joosten et al., "The janus face of Bartonella quintana recognition by toll-like receptors (TLRs): a review," European Cytokine Network, vol. 19, no. 3, pp. 113-118, 2008.

[21] P. A. Kyme, A. Haas, M. Schaller, A. Peschel, J. Iredell, and V. A. J. Kempf, "Unusual trafficking pattern of Bartonella henselaecontaining vacuoles in macrophages and endothelial cells," Cellular Microbiology, vol. 7, no. 7, pp. 1019-1034, 2005.

[22] W. Vermi, F. Facchetti, E. Riboldi et al., "Role of dendritic cellderived CXCL13 in the pathogenesis of Bartonella henselae Brich granuloma," Blood, vol. 107, no. 2, pp. 454-462, 2006.

[23] S. Resto-Ruiz, A. Burgess, and B. E. Anderson, "The role of the host immune response in pathogenesis of Bartonella henselae," DNA and Cell Biology, vol. 22, no. 6, pp. 431-440, 2003.

[24] A. M. McCord, S. I. Resto-Ruiz, and B. E. Anderson, "Autocrine role for interleukin-8 in Bartonella henselaeinduced angiogenesis," Infection and Immunity, vol. 74, no. 9, pp. 5185-5190, 2006. 
[25] N. G. Papadopoulos, D. Gourgiotis, A. Bossios, A. Fretzayas, M. Moustaki, and T. Karpathios, "Circulating cytokines in patients with cat scratch disease," Clinical Infectious Diseases, vol. 33, no. 6, pp. e54-e56, 2001.

[26] P. Glynn, R. Coakley, I. Kilgallen, N. Murphy, and S. O’Neill, "Circulating interleukin 6 and interleukin 10 in community acquired pneumonia," Thorax, vol. 54, no. 1, pp. 51-55, 1999.

[27] T. Musso, R. Badolato, D. Ravarino et al., "Interaction of Bartonella henselae with the murine macrophage cell line J774: infection and proinflammatory response," Infection and Immunity, vol. 69, no. 10, pp. 5974-5980, 2001.

[28] M. F. Minnick and J. M. Battisti, "Pestilence, persistence and pathogenicity: infection strategies of Bartonella," Future Microbiology, vol. 4, no. 6, pp. 743-758, 2009.

[29] L. Chiaraviglio, S. Duong, D. A. Brown, R. J. Birtles, and J. E. Kirby, "An immunocompromised murine model of chronic Bartonella infection," American Journal of Pathology, vol. 176, no. 6, pp. 2753-2763, 2010.

[30] R. Santos, O. Cardoso, P. Rodrigues et al., "Bacillary angiomatosis by Bartonella quintana in an HIV-infected patient," Journal of the American Academy of Dermatology, vol. 42, no. 2, part 1, pp. 299-301, 2000.

[31] M. C. Schmid, F. Scheidegger, M. Dehio et al., "A translocated bacterial protein protects vascular endothelial cells from apoptosis," PLoS Pathogens, vol. 2, no. 11, p. e115, 2006.

[32] C. C. Chang, Y. J. Chen, C. S. Tseng et al., "A comparative study of the interaction of Bartonella henselae strains with human endothelial cells," Veterinary Microbiology, vol. 149, no. 1-2, pp. 147-156, 2011.

[33] R. Chetty and R. M. Sabaratnam, "Upper gastrointestinal bacillary angiomatosis causing hematemesis: a case report," International Journal of Surgical Pathology, vol. 11, no. 3, pp. 241-244, 2003.

[34] P. Hofman, H. Raspaldo, J. F. Michiels, G. Garnier, and J. Santini, "Bacillary angiomatosis of the oral cavity in AIDS. A differential diagnosis of mucosal Kaposi's sarcoma," Revue de Stomatologie et de Chirurgie Maxillo-Faciale, vol. 94, no. 6, pp. 375-378, 1993.

[35] S. L. de Blanc, R. Sambuelli, F. Femopase et al., "Bacillary angiomatosis affecting the oral cavity. Report of two cases and review," Journal of Oral Pathology and Medicine, vol. 29, no. 2, pp. 91-96, 2000.

[36] S. R. Long, M. J. Whitfeld, C. Eades, J. E. Koehler, A. P. Korn, and C. J. Zaloudek, "Bacillary angiomatosis of the cervix and vulva in a patient with AIDS," Obstetrics and Gynecology, vol. 88, no. 4, part 2, pp. 709-711, 1996.

[37] M. Sala, B. Font, I. Sanfeliu, M. Quesada, I. Ponts, and F. Segura, "Bacillary angiomatosis caused by Bartonella quintana," Annals of the New York Academy of Sciences, vol. 1063, pp. 302-307, 2005.

[38] H. Bachelez, E. Oksenhendler, C. Lebbe et al., "Bacillary angiomatosis in HIV-infected patients: report of three cases with different clinical courses and identification of Rochalimaea quintana as the aetiological agent," British Journal of Dermatology, vol. 133, no. 6, pp. 983-989, 1995.

[39] B. Lienhardt, S. Irani, A. Gaspert, D. Weishaupt, and A. Boehler, "Disseminated infection with Bartonella henselae in a lung transplant recipient," Journal of Heart and Lung Transplantation, vol. 28, no. 7, pp. 736-739, 2009.

[40] V. R. Dharnidharka, G. A. Richard, R. E. Neiberger, and R. S. Fennell III, "Cat scratch disease and acute rejection after pediatric renal transplantation," Pediatric Transplantation, vol. 6, no. 4, pp. 327-331, 2002.
[41] K. R. Thudi, J. T. Kreikemeier, N. J. Phillips, P. R. Salvalaggio, D. J. Kennedy, and P. H. Hayashi, "Cat scratch disease causing hepatic masses after liver transplant," Liver International, vol. 27, no. 1, pp. 145-148, 2007.

[42] A. Humar and I. Salit, "Disseminated Bartonella infection with granulomatous hepatitis in a liver transplant recipient," Liver Transplantation and Surgery, vol. 5, no. 3, pp. 249-251, 1999.

[43] H. Bonatti, J. Mendez, I. Guerrero et al., "Disseminated Bartonella infection following liver transplantation," Transplant International, vol. 19, no. 8, pp. 683-687, 2006.

[44] A. M. Apalsch, B. Nour, and R. Jaffe, "Systemic cat-scratch disease in a pediatric liver transplant recipient and review of the literature," Pediatric Infectious Disease Journal, vol. 12, no. 9, pp. 769-774, 1993.

[45] D. H. Spach, A. S. Kanter, M. J. Dougherty et al., "Bartonella (Rochalimaea) quintana bacteremia in inner-city patients with chronic alcoholism," The New England Journal of Medicine, vol. 332, no. 7, pp. 424-428, 1995.

[46] M. Drancourt, J. L. Mainardi, P. Brouqui et al., "Bartonella (Rochalimaea) quintana endocarditis in three homeless men," The New England Journal of Medicine, vol. 332, no. 7, pp. 419423, 1995.

[47] P. Houpikian and D. Raoult, "Blood culture-negative endocarditis in a reference center: etiologic diagnosis of 348 cases," Medicine, vol. 84, no. 3, pp. 162-173, 2005. 


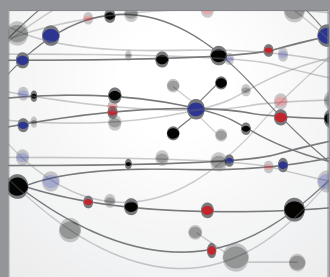

The Scientific World Journal
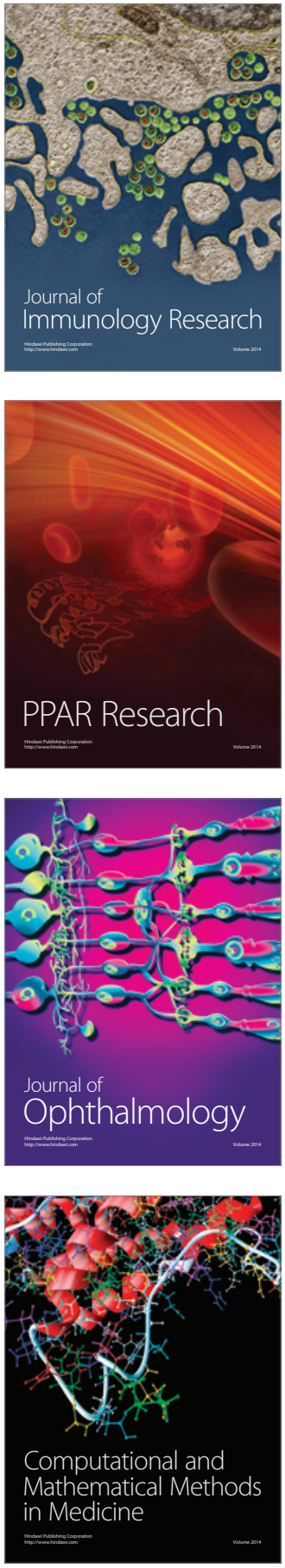

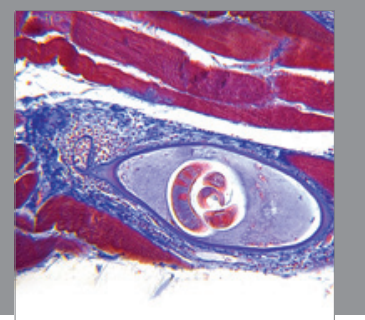

Gastroenterology

Research and Practice
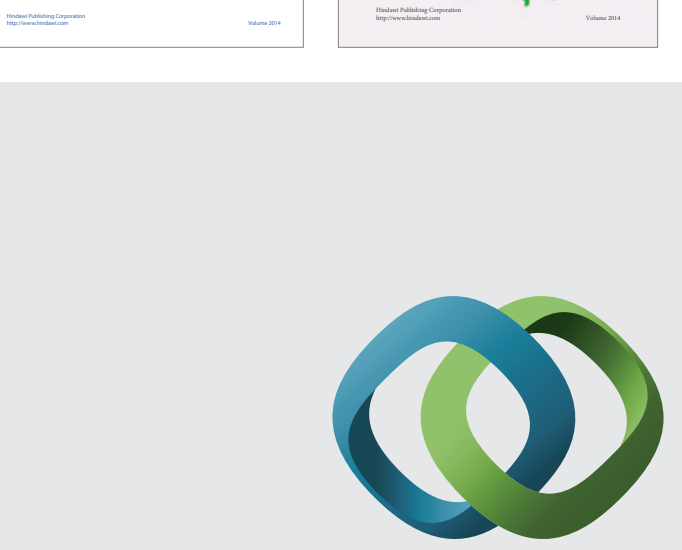

\section{Hindawi}

Submit your manuscripts at

http://www.hindawi.com
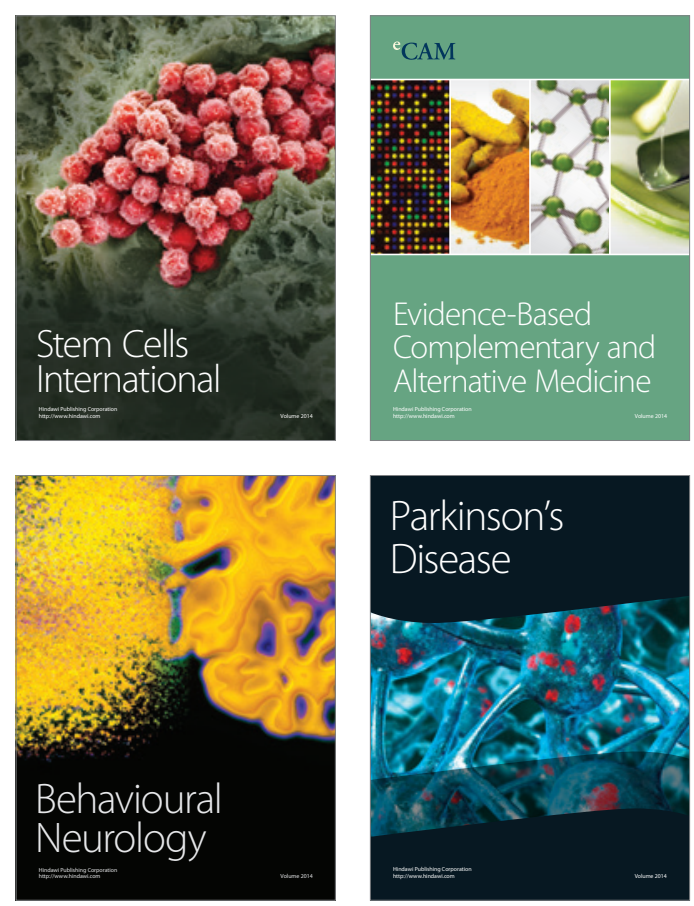

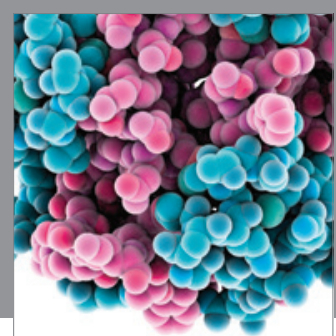

Journal of
Diabetes Research

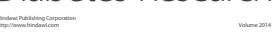

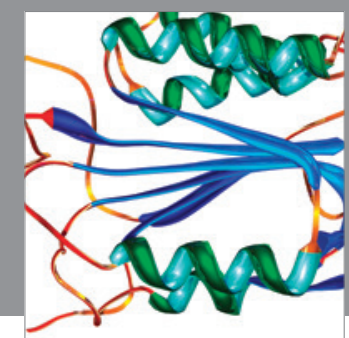

Disease Markers
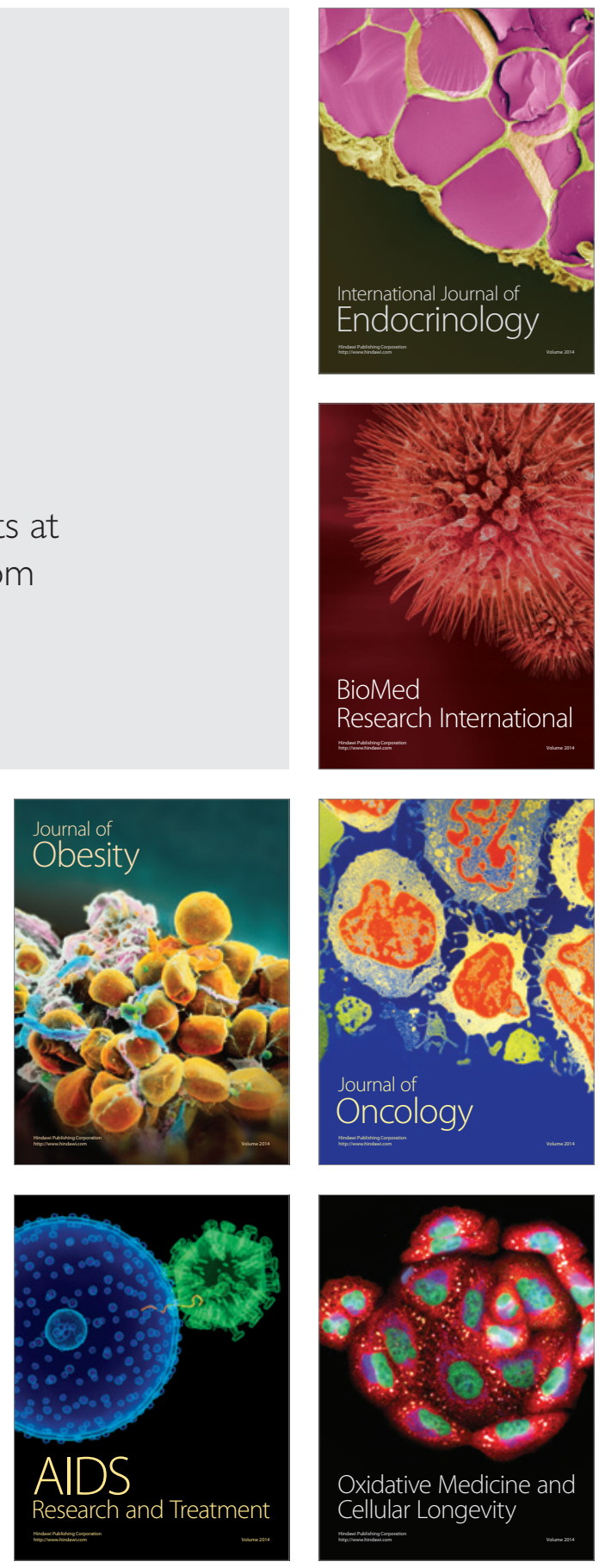